\title{
The Factor Structure of Cybersickness
}

\author{
Susan Bruck ${ }^{\mathrm{a}}$, Paul A. Watters ${ }^{\mathrm{b}}$ \\ a Department of Computing, Macquarie University, NSW 2109, Australia \\ ${ }^{b}$ Graduate School of Information Technology and Mathematical Sciences, University of Ballarat, Victoria 3353, Australia
}

\begin{abstract}
Cybersickness embraces a range of clinical symptoms reported in response to simulated motion in a computer generated, virtual reality environment. The Simulator Sickness Questionnaire (SSQ) has been the standard tool for measuring observed responses; however, many of the observed SSQ variables are highly correlated, so it is not clear which ones are appropriate to use as a basis for building an explanatory model. In this study, responses to the SSQ were analyzed using principal components analysis, and four significant factors - General Cybersickness, Vision, Arousal and Fatigue - were identified. An initial interpretation of these factors is provided in the context of a broader cybersickness model, with a view to constructing a new questionnaire with fewer, more focused questions.
\end{abstract}

Keywords: Simulator Sickness Questionnaire, virtual reality, factor analysis, Cybersickness,

\section{Introduction}

Therapeutic anxiety treatments, vocational training for pilots, astronauts and car drivers, often make use of a computer generated immersive virtual reality environment (VRE). These environments are widely accepted as safe and incrementally controllable [1, 2]. However, despite the recent increase in the use of VRE for learning and teaching, it is reported that cybersickness affects between $60-80 \%$ of users $[3,4]$. Cybersickness describes a collection of symptoms in which the individual who is exposed to a VRE reports symptoms akin to classical motion sickness, without the presence of physical motion [5]. Symptoms of cybersickness are expected if there is sufficient illusion of self movement (vection) [6, 7, 8]. Moreover, the literature reveals that an extended duration of exposure results in a greater severity of symptoms [9, 10]. Kennedy [9] reported that exposure duration between one and three hours, and repeated exposures are linearly related to the Simulator Sickness Questionnaire (SSQ) [11] responses in flight simulators. Notably, Kennedy [12] reports significant visually induced motion sickness symptoms as rated by the SSQ [11] responses in just 15 minutes. Indeed, an increase in symptoms in response to VRE exposure has been reported in as little as six [13] to ten minutes [3].

The SSQ [11] is a customized edition of the Pensacola Motion Sickness Questionnaire [14], whereby the original list of motion sickness symptoms is condensed to accommodate symptoms specific to simulator technology. The SSQ [11] requires the user to subjectively report the severity of symptoms such as general discomfort, fatigue, headache, eyestrain, difficulty in focusing, salivation, sweating, nausea, difficulty in

Email addresses: $\quad$ sbruck@science.mq.edu.au (Susan Bruck), p.watters@ballarat.edu.au ( Paul A. Watters) concentrating, fullness of head, blurred vision, dizziness, vertigo, stomach awareness or burping on a scale ranging from no symptoms through to severe symptoms.

Although the purpose of the SSQ [11] was initially to identify simulators that induce simulator sickness symptoms in the trainees, it is now accepted as a validated tool for assessing client responses in the research and treatment of psychiatric and psychological conditions [15, 16], occupational training scenarios $[17,18]$ as well as anti-motion sickness drug efficacy in a virtual environment [19]. We chose to use the SSQ [11] as a reliable tool for assessing symptomatic responses to our simulated environment.

A potential confound with the SSQ [11] is that it contains many variables that are highly correlated $[11,20]$. Kennedy [11] acknowledged this issue and provided a factor analytic model which identified three orthogonal groups of symptoms - oculomotor, disorientation and nausea - as a way of scoring the questionnaire. Another study [20] that examined reducing the number of questions of the SSQ [11] assessed a cohort of 164 clinically diagnosed anxiety disorder patients and 207 healthy individuals, using a principal factor analysis, reported that only two factors - oculomotor and nausea - were significant (Cronbachs alpha 0.87). Likewise, a regression model [21] was used to identify four SSQ [11] symptoms - headache, difficulty in concentrating, fullness of head and burping - to predict cybersickness. For this reason, we propose that a subset of the SSQ [11] is likely to be sufficient to determine whether a user is likely to experience cybersickness or not.

Anxiety has also been reported to correlate with significant movement within a VRE $[22,23]$. In addition to the [11] selfreport questionnaire, a range of symptomatic assessment options are available for measuring physiological responses to VREs based on autonomic nervous system function. Although some studies have suggested anxiety is the response measured, 
anxiety is a term that implies psychological stress and suggests an element of aversion to the stimuli [24]. A more accurate definition would be the term arousal whereby we refer to a state of activation.

The Yerkes-Dodson Law [25] hypothesized that increased arousal improves performance up to an optimal point, where after, a further increase in arousal results in a decreased performance, a feature known as the inverted U principle. Within a research context, engaging physiological responses may offer an objective measure. Cardiac activity is a robust measure of performance arousal [26, 27, 28, 29]. Increased cardiac function has been reported to be correlated with visually induced motion sickness (VIMS) within virtual environments [30, 31]. Specifically, heart rate is more sensitive to varied cognitive demands than heart rate variability, and typically increases in response to higher mental load [29, 32].

The respiratory system maintains blood oxygen and carbon dioxide concentrations within specific values. At rest a normal person breathes approximately 12 times per minute. Increasing physical activity stimulates an increase in depth and breathing rate. Similarly, intense mental effort, or psychological stress, even in the absence of physical activity can increase arousal levels and cause an increased rate and depth of respiration - hyperventilation. Breathing rate variability is a convenient and useful measure of mental load and stress [33] and is considered a sensitive indicator of intense concentration [34]. Respiratory change is also a sensitive index of anxiety [35], and is a reliable and valid noninvasive measure of hyperventilation in phobic and control participants [36]. Hyperventilation has also been reported to alleviate the symptoms of nausea in response to a rotating optokinetic drum [37], an oscillating television monitor or rotating and tilting physical environments $[38,39,40]$. In this study we propose investigating whether respiration changes in response to considerable movement within a VRE [41, 22, 21] could be an underlying physiological catalyst for cybersickness.

Engaging physiological methods may offer an objective appraisal of cybersickness within a research context in order to establish fundamental data; one limitation is, however, measuring physiological responses requires substantial administrator technical expertise. The adherence of electrodes to the client is invasive, time consuming and necessitates specialized electrocardiographic (ECG) equipment, thereby illustrating the need for a straightforward assessment tool. By identifying the underlying factors that activate the symptoms assessed by the SSQ [11], we propose these factors will provide an insight into the physiological source of cybersickness, and assist in the development of a comprehensive theory of cybersickness. Cybersickness measures are generally administered (a) during a VRE session, (b) immediately afterwards, or (c) in order to measure the difference between pre-exposure and post-exposure test results. Yet it is not clear how predictive these measures are in identifying the susceptibility of an individual to any particular symptom. As Stroffregen and Riccio [42] stated, one of the main problems in predicting cybersickness is the complexity of the nervous system and the limited understanding of how sensory conflict [41] between visual input and cybersickness symp- toms interplay. We believe there is a strong need for a simple, non-invasive and economical instrument capable of determining a client's susceptibility to cybersickness in a VRE. The four core requirements we have identified are:

- Must predict cybersickness susceptibility when exposed to computer generated immersive virtual reality environments

- Must be straightforward for users to respond

- Needs to identify critical indicators of cybersickness

- Must provide data in an easy-to-analyze form

In order to identify the elements that are most likely to be predictive for cybersickness (dependent variable), we propose using a factor analysis on the independent variable results from (a) an SSQ [11] administration, (b) an anxiety questionnaire [22], (c) respiration (breaths per minute) and (d) cardiac activity (ECG; beats per minute), to determine whether there are a limited number of latent variables that may be key indicators of cybersickness symptoms in response to a VRE. The data to be analyzed comes from a previously reported experiment [23] in which a significant effect for simulated motion was observed between a control and experimental condition for cybersickness. Thus, the goal of this work is not to look a differences between control and experimental groups, but to understand what factors underlie the significance of the experimental group.

\section{Methods}

As reported in [23], volunteer university undergraduates aged between 18 and 30 years of age participated in this experiment. The sample cohort (18 male and 10 female) were healthy and had normal or corrected to normal vision. Participants had no prior experience in the university VRE. Prior to the experiment, the participants received an email package with a Health Questionnaire, Ethics Consent Form and information letter requiring the participant to refrain from eating or drinking for two hours prior to the experiment, and not to consume illicit drugs or caffeine for 12 hours prior to the experiment.

The participants were fluent in written and spoken English. Ethics approval for the study was obtained from the Macquarie University Human Ethics Committee. The Ethics Consent Form explained that the aim of the experiment was to investigate motion sickness in a virtual environment. Participants were advised that they had the right to discontinue the experiment at any time. No familiarization took place - all participants were naive to the VRE and the laboratory.

The experiment was conducted using a within-subject design to compare responses in a control condition (VRE with low simulated motion) and an experimental condition (VRE with high simulated motion). Participants acted as their own controls. Participants completed an Anxiety Scale [22] prior to the experiment, then again after the control and the experimental conditions. The Anxiety Scale [22] is a self rating tick box questionnaire with six questions, No Symptoms, Some Anxiety, Mild Anxiety, Moderate Anxiety, Severe Anxiety, Extreme 
Anxiety which are scored between 0 and 5. The SSQ [11] was also completed prior to commencing the experiment and again after both experimental and control conditions.

To record respiration and ECG data we used the Biopac Systems Inc. MP35 data acquisition unit. Respiration data was recorded by placing the respiratory transducer around the participants chest, below the armpits and above the nipples whilst ensuring a slight tight tension was felt at maximal expiration. The transducer was worn over a light shirt or top. ECG data was recorded by placing electrodes on the right anterior forearm wrist, left leg just above the medial malleous (inner ankle) and right leg just above the medial malleous (ground electrode). Calibration procedures were followed as per the Biopac Instruction Manual [43].

The VREs were displayed on a parabola curved 160 degrees field of view projection screen $(6.2 \mathrm{~m} \times 1.7 \mathrm{~m})$. The participants viewed the VRE through Liquid Crystal Display synchronized shutter glasses that generate the three dimensional effect. The control condition presents a snow scene with gently rolling hills that the participant travels slowly over. In this scene there is little simulated movement other than the steady movement across the terrain (Figure 1). The experimental condition portrays a rollercoaster ride (Figure 2), whereby the participant travels along the sharp inclines and steep declines exposing the participant to moderate to severe simulated movement. Both the experimental and control conditions lasted for two minutes, and ran three circuits of the environment making a six minute journey. The participant was seated on a standard arm rest chair and was not restrained.

Only the results from the experimental condition were used in the current analysis, given the significant differences in cybersickness and anxiety between control and experimental conditions that have been previously reported [23].

Figure 1: Control Condition (Snow Scene)

Figure 2: Experimental Condition (Rollercoaster)

\section{Results}

Results from the SSQ [11] and Anxiety Questionnaire [22], respiratory rate (breaths per minute) and heart rate (beats per minute) were collected from the experimental condition and were tabulated and analyzed using the statistical package STATA Release 11. For both the respiratory rate and the heart rate we analyzed the data from the final one minute of the sample [22]. Factor routine, with principal components analysis and no rotation was chosen to analyze the data. Using the principal component analysis we applied a co-efficient matrix on the dependent variables. The factor analysis was performed to identify the latent structures within the set of eighteen variables. By using this analysis we were able to reduce our data to identify a smaller number of factors that are likely to be creating the observed cybersickness symptoms.
The results obtained are shown in Table 1. Factors were only retained for variable loadings, where the factor eigenvalue was greater than 1 (i.e., using the Kaiser Criterion [44]). Table 2 shows the factor table, the result of a factor analysis of the SSQ [11] plus anxiety, respiration and cardiac activity (ECG). The eighteen scales appear down the left column and the four factors extracted appear across the top of the table. The body of the table provides the loadings of each of the factors. Loadings greater than 0.30 are considered to be a significantly strong contribution to the factor. Table 3 provides the descriptive statistics. Four factors were thus identified, cumulatively accounting for $78.27 \%$ of the variance observed. When we consider that the cumulative variance for only four factors was greater than $78 \%$ we were keen to consider the structure of the correlations, and the underlying physiological processes impacting upon the set of variables. In the following subsections, a preliminary interpretation of each of the four factors is provided.

\subsection{General Cybersickness Factor}

This factor has positive-only loadings for a range of the most common symptoms associated with cybersickness, including cardiac activity, general discomfort, fatigue, salivation, difficulty in focusing, increased sweating, increased nausea, difficulty in concentrating, fullness of head, dizziness, vertigo, stomach awareness, burping and increased anxiety. In short, this single underlying symptomatic factor accounts for $38.75 \%$ of the variability of eighteen variables, suggesting that the symptoms of this factor are most likely to be combining to create the sensation of nausea and a general unwellness.

\subsection{Vision Factor}

This factor contains only three extra elements in addition to the variables that loaded significantly in the first factor: respiration, headache and eyestrain. We believe that this factor displays the relationship between the direct sensory input (eyestrain) and headaches that are reported by participants who are exposed to high levels of movement within a simulated environment. The affinity between the physical symptoms of eyestrain and headache in response to computerized visual display units [45], and simulator training environments [46] are well documented and we propose that this tendency to headaches may be associated with increased excitability of the visual cortical areas of the brain [47]. We suggest that this factor provides the link between general cybersickness and the simulated motion, whereby the sensory input is provided through the visual system, which can then become overloaded and consequently induce a headache. It is interesting to note that anxiety is not associated with this factor; thus, whilst general cybersickness is associated with anxiety, the visual factor is not.

\subsection{Arousal Factor}

The arousal factor sees respiration exclusively being associated with difficulty focusing and concentrating, blurred vision, vertigo and stomach awareness. Respiration is a key measure of ANS activity, as evidenced by changes in blood levels of carbon dioxide in response to exercise or psychological anxiety 


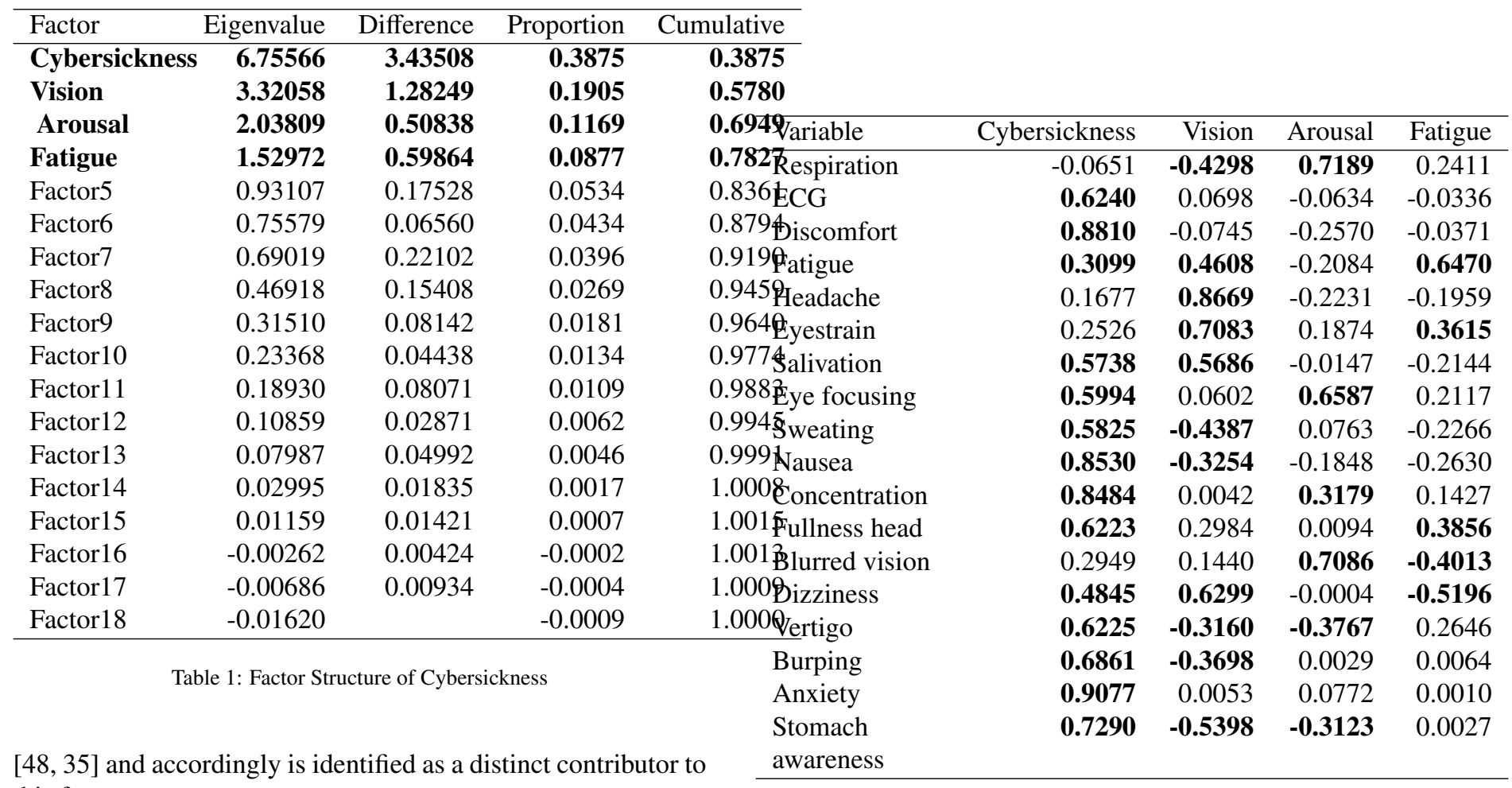

this factor.

Table 2: Factor Loadings for Cybersickness

\subsection{Fatigue Factor}

The final factor identifies some symptoms associated with fatigue, such as fullness of head and eyestrain, with self-reported fatigue. Blurred vision and dizziness also loaded highly on this factor. This factor contributed the least amount of variance in the model, but presumably for longer periods than 6 minutes of testing, it would take on greater significance.

\subsection{Discussion}

The motivation for applying a principal components analysis to the set of variables which included the symptoms of the SSQ [11], respiration, cardiac and anxiety data, was that we were interested in determining which variables would form a coherent subset of factors that could be used to develop an objective test for measuring a range of symptoms that are reported by participants in VREs. The goal was to reduce the number of variables required to be assessed into fewer factors. These factors are able to concentrate the relationships between the variables into a more efficient way of evaluating the unpleasant responses reported after immersion in a VRE. We have successfully achieved this goal. The results of this study suggest that cybersickness has four significant, latent components that could be more directly assessed possibly by a combination of self-report and physiological measures:

- General cybersickness

\begin{tabular}{llllr}
\hline Variable & Mean & Std.Dev. & Min. & Max \\
\hline Respiration & 11.57143 & 7.77188 & 0 & 21 \\
ECG & 57.35714 & 35.46016 & 0 & 105 \\
Discomfort & 0.9642857 & 0.8811669 & 0 & 3 \\
Fatigue & 0.4642857 & 0.6372477 & 0 & 2 \\
Headache & 0.25 & 0.5181877 & 0 & 2 \\
Eyestrain & 0.7142857 & 0.6586823 & 0 & 2 \\
Salivation & 0.4285714 & 0.8357109 & 0 & 3 \\
Eye focusing & 0.6428571 & 0.8261596 & 0 & 2 \\
Sweating & 0.6428571 & 1.026114 & 0 & 3 \\
Nausea & 0.7857143 & 0.9946949 & 0 & 3 \\
Concentration & 0.5714286 & 0.790151 & 0 & 3 \\
Fullness head & 0.5357143 & 0.6929349 & 0 & 2 \\
Blurred vision & 0.2857143 & 0.5345225 & 0 & 2 \\
Dizziness & 0.3928571 & 0.7859547 & 0 & 3 \\
Vertigo & 0.25 & 0.5853141 & 0 & 2 \\
Burping & 0.25 & 0.5853141 & 0 & 2 \\
Anxiety & 0.9642857 & 1.070899 & 0 & 3 \\
Stomach & 0.6428571 & 0.8698355 & 0 & 3 \\
awareness & & & & \\
\hline
\end{tabular}

- Vision

Table 3: Descriptive Statistics

- Arousal

- Fatigue 
The General Cybersickness Factor accounted for $38.75 \%$ of the variability of the analyzed variables. This result suggests that it is the common physiological processes underlying the fourteen symptoms that are causing a general unwellness in the participants. The affinity between the sensory input from exposure to the VRE and the increase in arousal and the consequential changes in respiration rate seems to distinguish respiration as a latent structure underpinning cybersickness. It is plausible that the underlying physiological initiator, hyperventilation, is the result of an increase in arousal in response to the high levels of movement within the VRE.

Anxiety is a psychological and physiological state of arousal that presents in individuals in response to particular stimuli [24]. Anxiety has been reported in response to immersive VREs that have high levels of movement on the screen [22, 23] and demonstrated to be correlated with increase in severity of several SSQ [11] symptoms [21]. The ANS responds to anxiety in several ways. One of the primary responses to a stressor is a change in respiration rate $[35,48]$, and this variation in breathing depth and frequency impacts on the levels of carbon dioxide in the blood.

The study of the effect of changes in carbon dioxide levels in cerebral blood flow has recently become an important research area. Recent technological advances using fMRI has shown the association between anxiety disorders and respiratory alterations in arterial carbon dioxide [35]. The relationship between breathing rate changes specifically, hyperventilation (increased rate and depth of breathing) and panic attack symptoms are well documented [36, 49]. Hyperventilation produces changes in cerebral blood flow which leads to feelings of lightheadedness, dizziness and concentration problems [50] in response to a reduction in carbon dioxide levels. By comparison, panic attack symptoms in the context of Anxiety Disorder [51] include heart palpitations, breathing difficulties, nausea, lightheadedness and dizziness.

The fourth factor we identified was Fatigue.This finding is in line with previous studies which demonstrate the association between drowsiness and simulated movement $[52,53,54]$. Fatigue has previously been reported to increase in a controlled study, in response to considerable simulated motion in a VRE [31]. The variables that loaded highly in this factor along with fatigue were eyestrain, blurred vision, dizziness and fullness of head. The relationship between rapid eye movements (nystagmus) the resultant eye muscle fatigue, and headache and migraine symptoms $[55,56,57,58,59]$ is well documented. In fact, the Eye Movement Theory of motion sickness [60] supports the notion that over stimulation of the muscles of the eye as a result of specific eye movements within a virtual environment can lead to headache and eye muscle fatigue.

Having demonstrated that there are four latent factors underlying cybersickness in an immersive VRE using a considerable level simulated motion, the key question is whether we can generalize these results to other virtual environments, and/or to other environments such as computer gaming? In order to establish whether these results are generalizable, firstly, we would need to compare the results from this study with an equivalent study undertaken in a non-immersive gaming environment.
Secondly, we could construct a new form of SSQ [11] with a reduced number of scales to try and more accurately measure the latent factors directly. Finally, since the current VRE does not require any user interaction or control, it is possible that the factor structure we have observed would be impacted if the user had control of movement and speed within the VRE. In further studies, it would be worthwhile investigating whether providing an option for the client to regulate the virtual movement within the VRE and whether this control would demonstrate a decrease in arousal as a result of reduced anxiety.

The well known phenomenon of the Yerkes-Dodson Law [25], indicates that an increase ANS arousal in response to a perceived stress affects an individuals ability to concentrate effectively beyond a critical threshold [61]. As in the YerkesDodson Law [25], the changes in respiration rate which occur in response to anxiety as a response to stimuli of high movement virtual environments, appear to be in turn creating changes in carbon dioxide blood levels which in turn are producing symptoms that are being recognised as increases in anxiety, thus reducing the participants ability to concentrate effectively on the content of the environment. Further research is required to determine how these different components fit into an overall explanatory model. Certainly, a single experiment is not sufficient to do this, for example, future studies could investigate longer periods of immersion, and its impact on fatigue.

An important requirement for any form of vocational or training exercise necessitates an easy to administer assessment tool for evaluating an individuals susceptibility to unpleasant side effects. It is therefore, necessary for a screening or assessment tool to be straightforward for client responses, as well as simple for the administrator to analyze the results.

Finally, the four factors identified provide further insight into the underlying causes of cybersickness. In identifying these factors our attention is drawn back to the need for a comprehensive theory of cybersickness. Our theory that it is an increase in arousal (anxiety) that promotes changes in respiration rate, which in turn generates reduced carbon dioxide levels in cerebral blood flow. This lowering of carbon dioxide results in lightheadedness [50], which subsequently presents as symptoms of dizziness, fatigue, difficulty in concentrating, feelings of dissociation (fullness of head [11]) and associated anxiety. It is these symptoms which cue the individual that their body is under stress [50].

In conclusion, in classifying the four factors, we have identified the latent structures that create the physiological responses that cause anxiety and cybersickness in a VRE with considerable movement. The consequences for individuals who are exposed to high levels of simulated movement have been shown to be significant in as little as six minutes $[31,21]$. In summary, we have demonstrated that with considerable simulated movement in an immersive environment; where the participant is not in control of the environment, increase in arousal and changes in respiration rate create a modus operandi that initiates physiological changes to carbon dioxide levels and changes in perceived wellness. Further experiments will shed led light on this important issue. 
[1] T. D. Parsons, A. A. Rizzo, Affective outcomes of virtual reality exposure therapy for anxiety and specific phobias: A meta-analysis, Journal of Behavior Therapy and Experimental Psychiatry 39 (3) (2008) 250-261.

[2] M. B. Powers, P. M. G. Emmelkamp, Virtual reality exposure therapy for anxiety disorders: A meta-analysis, Journal of Anxiety Disorders 22 (3) (2008) 561-569.

[3] S. V. G. Cobb, S. Nichols, A. Ramsey, J. R. Wilson, Virtual realityinduced symptoms and effects (VRISE), Presence: Teleoperators \& Virtual Environments 8 (2) (1999) 169-186.

[4] E. Regan, K. Price, The frequency and occurrence and severity of sideeffects of immersion in virtual reality, Aviation space and environmental medicine 65 (6) (1994) 527-530.

[5] E. McCauley, Michael, J. Sharkey, Thomas, Cybersickness: perception of self-motion in virtual environments, Presence 1 (3) (1992) 311-318.

[6] E. Miller, A. Graybiel, Motion sickness produced by head movement as a function of rotational velocity, Aerospace Medicine 1 (13) (1970) 311318.

[7] K. E. Money, Motion sickness, Physiol. Rev. 50 (1) (1970) 1-39.

[8] L. J. Hettinger, K. S. Berbaum, R. Kennedy, W. P. Dunlap, M. D. Nolan, Vection and simulator sickness, Military Psychology 2 (3) (1990) 171181.

[9] R. Kennedy, K. M. Stanney, W. Dunlap, Duration and exposure to virtual environments: Sickness curves during and across sessions, Presence: Teleoperators \& Virtual Environments 9 (5) (2000) 463-472.

[10] J. R. Lackner, R. S. Larry, Motion sickness, in: Encyclopedia of Neuroscience, Academic Press, Oxford, 2009, pp. 989-993.

[11] R. Kennedy, N. Lane, K. Berbaum, M. Lilienthal, Simulator Sickness Questionnaire: An enhanced method for quantifying simulator sickness, International Journal of Aviation Psychology 3 (3) (1993) 203-220.

[12] R. Kennedy, J. M. Drexler, R. Kennedy, Research in visually induced motion sickness, Applied Ergonomics 41 (4) (2010) 494-503.

[13] P. A. Howarth, S. Clemes, Susceptibility to induced visual discomfort during menstrual cycle while viewing a visual display unit, Optometry and vision science: official publication of the American Academy of Optometry 83 (3) (2006) 190-194.

[14] R. Kellogg, R. Kennedy, A. Graybiel, Motion sickness symptomatology of labyrinthine defective and normal subjects during zero gravity maneuvers, Aerospace Medicine 36 (1965) 315-318.

[15] D. Freeman, K. Pugh, N. Vorontsova, A. Antley, Testing the continuum of delusional beliefs, Journal of Abnormal Psychology 119 (1) (2010) 83-92.

[16] G. Robillard, S. Bouchard, T. Fournier, P. Renaud, Anxiety and presence during VR immersion: A comparative study of the reactions of phobic and non-phobic participants in therapeutic virtual environments derived from computer games, CyberPsychology \& Behavior 6 (5) (2003) 467476.

[17] J. Y. Chen, C. Joyner, Concurrent performance of gunner's and robotics operator's tasks in a multitasking environment, Military Psychology 21 (1) (2009) 98-113

[18] D. M. Flinton, N. White, Preliminary findings on the virtual environment for radiotherapy training VRET system: Simulator sickness and presence, Journal of Radiotherapy in Practice 8 (04) (2009) 169-176.

[19] C. Regan, An investigation into nausea and other side-effects of headcoupled immersive virtual reality, Virtual Reality 1 (1) (1995) 17-31.

[20] S. Bouchard, G. Robillard, P. Renaud, Revising the factor structure of the Simulator Sickness Questionnaire, Annual Review of CyberTherapy and Telemedicine 5 (Summer) (2007) 128-137.

[21] S. Bruck, P. A. Watters, Autonomic nervous system factors underlying anxiety in virtual environments: A regression model for cybersickness, International Conference on Virtual Systems and MultiMedia (2009) 6772doi:http://doi.ieeecomputersociety.org/10.1109/VSMM.2009.16.

[22] Y. Y. Kim, H. J. Kim, E. N. Kim, H. D. Ko, H. T. Kim, Characteristic changes in the physiological components of cybersickness, Psychophysiology 42 (5) (2005) 616-625.

[23] S. Bruck, P. Watters, Cybersickness and anxiety during simulated motion Implications for VRET, in: G. R. Brenda K. Wiederhold (Ed.), Studies in Health Technology and Informatics Annual Review of Cybertherapy and Telemedicine 2009 - Advanced Technologies in the Behavioral, Social and Neurosciences, Vol. 144, IOS Press, 2009, pp. 169-173.

[24] G. Dieleman, J. van der Ende, F. Verhulst, A. Huizink, Perceived and physiological arousal during a stress task: Can they differentiate between anxiety?, Psychoneuroendocrinology 35 (2010) 1223-1234.

[25] R. Yerkes, J. Dodson, The relationship of strength of stimulus to rapidity of habit-formation, Journal of Comparative Neurology and Psychology 18 (1908) 459-482.

[26] P. Lehrer, M. Karavidas, S.-E. Lu, E. Vaschillo, B. Vaschillo, A. Cheng, Cardiac data increase association between self-report and both expert ratings of task load and task performance in flight simulator tasks: An exploratory study, International Journal of Psychophysiology 76 (2) (2010) 80 - 87. doi:DOI: 10.1016/j.ijpsycho.2010.02.006.

[27] M. Meehan, S. Razzaque, B. Insko, M. Whitton, F. P. Brooks Jr, Review of four studies on the use of physiological reaction as a measure of presence in stressfulvirtual environments, Applied Psychophysiology \& Biofeedback 30 (3) (2005) 239-258.

[28] C. Gemmill, Physiology in Aviation, Thomas, Oxford, England, 1943.

[29] G. Wilson, An analysis of mental workload in pilots during flight using multiple psychophysiological measures, The International Journal of Aviation Psychology 12 (1) (2002) 3-18.

[30] Y. Yokota, M. Aoki, K. Mizuta, Y. Ito, N. Isu, Motion sickness susceptibility associated with visually induced postural instability and cardiac autonomic responses in healthy subjects, Acta Oto-Laryngologica 125 (3) (2005) $280-285$.

[31] S. Bruck, P. Watters, Estimating cybersickness of simulated motion using the Simulator Sickness Questionnaire (SSQ): A controlled study, in: Sixth International Conference on Computer Graphics, Imaging and Visualization, CGIV, 2009, pp. $486-488$.

[32] J. Veltman, A. Gaillard, Physiological workload reations to increasing levels of task difficulty, Ergonomics 41 (5) (1998) 656-669.

[33] A. Roscoe, Assessing pilot workload. why measure heart rate, HRV and respiration?, Biological Psychology 34 (1992) 259-287.

[34] J. Eicheler, Telemetry of the heart rate and respiratory rate of gliding and motor drvien sports planes under different flight conditions (in German), Mschr Unfallheilk 78 (1975) 283-289.

[35] N. Giardino, S. Friedman, S. Dager, Anxiety, respiration, and cerebral blood flow: Implications for functional brain imaging, Comprehensive Psychiatry 48 (2) (2007) 103-112.

[36] G. Alpers, F. Wilhelm, W. Roth, Psychophysiological assessment during exposure in driving phobic patients, Journal of Abnormal Psychology 114 (1) (2005) 126-139.

[37] M. Jokerst, M. Gatto, R. Fazio, R. Stern, K. Koch, Slow deep breathing prevents the development of tachygastria and symptoms of motion sickness, Aviation Space and Environmental Medicine 70 (12) (1999) 11891192

[38] F. Yen Pik Sang, J. F. Golding, M. Gresty, Suppression of sickness by controlled breathing during mildly nauseogenic motion, Aviation Space and Environmental Medicine 70 (9) (2003) 998-1002.

[39] A. Mert, W. Bles, S. Nooij, Hyperventilation in a motion sickness desensitization program, Aviation, Space, and Environmental Medicine 78 (2007) 505-509

[40] A. Mert, I. Klopping-Ketelaars, W. Bles, Respiratory impact on motion sickness induced by linear motion, Annals of the New York Academy of Sciences 1164 (Basic and Clinical Aspects of Vertigo and Dizziness) (2009) 173-179.

[41] J. Reason, J. Brand, Motion Sickness, Academic Press, London, 1975.

[42] T. A. Stoffregen, G. E. Riccio, An ecological critique of the sensory conflict theory of motion sickness, Ecological Psychology 3 (3) (1991) 159 194.

[43] Biopac, Manual Part No. MANSL35, Biopac Systems Inc., 2006.

[44] H. F. Kaiser, The application of electronic computers to factor analysis, Educational and Psychological Measurement 20 (1) (1960) 141-151.

[45] T. Nakazawa, Y. Okubo, Y. Suwazono, E. Kobayashi, S. Komine, N. Kato, K. Nogawa, Association between duration of daily VDT use and subjective symptoms, American Journal of Industrial Medicine 42 (2002) 421426.

[46] J. O. Brooks, R. R. Goodenough, M. C. Crisler, N. D. Klein, R. L. Alley, B. L. Koon, W. C. L. Jr., J. H. Ogle, R. A. Tyrrell, R. F. Wills, Simulator sickness during driving simulation studies, Accident Analysis \& Prevention 42 (3) (2010) $788-796$

[47] M. Gerwig, L. Niehaus, O. Kastrup, P. Stude, H. C. Diener, Visual cortex excitability in migraine evaluated by single and paired magnetic stimuli, Headache 45 (10) (2005) 1394-1399.

[48] J. Laffey, B. Kavanagh, Hypocapnia, The New England Journal of 
Medicine 347 (2002) 43-53.

[49] R. Ley, Blood, breath, and fears; a hyperventilation theory of panic attacks and agoraphobia, Clinical Psychology Review 5 (4) (1985) 271-285.

[50] J. Bresseleers, I. Van Diest, S. De Peuter, P. Verhamme, O. Van den Bergh, Feeling lightheaded: The role of cerebral blood flow, Psychosomatic Medicine 72 (2010) 1-9.

[51] APA, Diagnostic and Statistical Manual of Mental Disorders, Fourth Edition (DSM-IV), American Psychiatric Association, 2000.

[52] A. Graybiel, J. Knepton, Sopite syndrome: A sometimes sole manifestation of motion sickness, Aviation Space and Environmental Medicine 47 (8) (1976) 873-882.

[53] E. Kiniorski, S. Weider, J. Finely, E. Fitzgerald, J. Howard, P. Di Nardo, L. Guzy, Sopite symptoms in the optokinetic drum, Aviation Space and Environmental Medicine 71 (10) (2004) 872-875.

[54] B. Lawson, A. Mead, The sopite syndrome revisited: Drowsiness and mood changes during real or apparent motion, Acta Astronaut 43 (1998) 181-192.

[55] G. Clement, S. Wood, M. F. Reschke, Effects of microgravity on the interaction of vestibular and optokinetic nystagmus in the vertical plane, Aviation Space and Environmental Medicine 63 (1992) 778-784.

[56] V. K. Gupta, Motion sickness is linked to nystagmus-related trigeminal brain stem input: a new hypothesis, Medical Hypotheses 64 (6) (2005) $1177-1181$.

[57] P. L. M. Huygen, W. I. M. Verhagen, E. J. J. M. Theunissen, M. G. M. Nicolasen, Compensation of total loss of vestibulo-ocular reflex by enhanced optokinetic response, Acta Oto-Laryngologica 108 (5 supp 468) (1989) $359-364$.

[58] R. Young, Laurence, H. Sienko, Kathleen, E. Lyne, Lisette, H. Hecht, A. Natapoff, Adaptation of the vestibulo-ocular reflex, subjective tilt, and motion sickness to head movements during short-radius centrifugation, Journal of Vestibular Research 13 (2) (2003) 65-77.

[59] W. Cheshire, Peripheral autonomic function and dysfunction, in: G. W. B. Appenzeller, P. J. Vinken (Ed.), The Autonomic Nervous System, Elsevier Health Sciences, Amsterdam, 2000, pp. 105-142.

[60] S. Ebenholtz, Motion sickness and oculomotor systems in virtual environments, Presence 1 (3) (1992) 302-305.

[61] P. A. Watters, F. Martin, Z. Schreter, Caffeine and cognitive performance: The nonlinear Yerkes-Dodson Law, Human Psychopharmacology: Clinical and Experimental 12 (3) (1997) 249-257. 